\title{
Dynamical Systems Theory and Explanatory Indispensability
}

\author{
Juha Saatsi*
}

I examine explanations' realist commitments in relation to dynamical systems theory. First I rebut an 'explanatory indispensability argument' for mathematical realism from the explanatory power of phase spaces. Then I critically consider a possible way of strengthening the indispensability argument by reference to attractors in dynamical systems theory. The take-home message is that understanding of the modal character of explanations (in dynamical systems theory) can undermine Platonist arguments from explanatory indispensability.

1. Introduction. Many scientific explanations use theoretical resources of highly contested ontological status. There are countless explanations that make essential use of idealizations, abstractions, and mathematics, for example. On the one hand, scientists typically adopt a broadly instrumentalist attitude toward these aspects of explanations. Even scientists of an ambitiously realist bent do not commit themselves uniformly to everything employed in scientific explanations. Many philosophers also regard the abstract and idealized features of explanations as playing a broadly instrumental role, even when they take explanations to latch onto unobservable reality in deeply explanatory ways.

On the other hand, others follow a broadly Quinean lead to issues of realism and ontological commitment and argue for realist commitment to mathematics or other contestable features of explanations on the grounds of their indispensable explanatory utility. So-called explanatory indispensability arguments have been advanced in this spirit with respect to mathematics, idealizations, abstractions, and emergence, for example (see, e.g., Baker 2009; Psillos 2010, 2011; Lyon 2012). It has been argued that realists' selective instrumentalism about such indispensable features of explanations is only a matter of an

\footnotetext{
*To contact the author please write to: Woodhouse Lane, Leeds LS2 9JT, United Kingdom; e-mail: J.T.Saatsi@leeds.ac.uk.
}

Philosophy of Science, 84 (December 2017) pp. 892-904. 0031-8248/2017/8405-0008\$10.00 Copyright 2017 by the Philosophy of Science Association. All rights reserved. 
unjustifiable bias for features of reality that are causally explanatory, serve a metaphysical reductionist ideology, or have clearly identifiable locations in space and time.

Various specific debates around explanatory indispensability exemplify this juxtaposition on a deep central issue: what are explanations' realist commitments? I believe that one of the key reasons these debates continue unabated is that they are often conducted (too) independently of a sufficiently clear sense of what scientific explanations amount to. The main message of this article is that the way forward is to conduct these debates in relation to sufficiently well-articulated philosophical accounts of explanation. This is a very broad thesis that I cannot hope to defend in general terms in a short article. Instead, I will support it by criticizing in this spirit a specific explanatory indispensability argument for mathematical realism in order to demonstrate the broader lesson.

Realist reasoning from explanatory indispensability varies in its details from case to case, but we can abstract away from these details and present the central line of thought in general terms. In the context of explanatorily indispensable mathematics, this yields a schematic explanatory indispensability argument (EIA; Baker 2009, 613):

(P1) We ought rationally to believe in the existence of any entity that plays an indispensable explanatory role in our best scientific theories.

(P2) Mathematical objects play an indispensable explanatory role in science.

(C) Hence, we ought rationally to believe in the existence of mathematical objects.

The main objective of this article is to bring out some unwarranted presuppositions of this line of thought. Thus presented, the gist of the problem is that P1 has very little going for it from the perspective of a well-motivated philosophical account of explanation. That is, given what we know about the nature of explanations, there are very good reasons not to adopt a blank commitment to any feature of reality that plays an indispensable explanatory role. Alternatively, we can take issue with P2: from said perspective, there is little point to speak bluntly of 'indispensable explanatory role' without further clarification as to the specific nature of that role. Given what we know about explanations, 'indispensable explanatory role' can mean different things, only some of which support realist commitment.

I will bring out these issues with EIA by focusing on a particular explanatory indispensability argument for mathematical Platonism that capitalizes on the explanatory power of phase spaces (Lyon and Colyvan 2007). Although this argument is often mentioned in the literature, it has hitherto escaped the right kind of critical attention. I will first present the argument and 
discuss its shortcomings from the perspective of a modal-dependence view of explanation, according to which explanations provide information about how the explanandum depends on the explanans (sec. 2). ${ }^{1}$ I will then consider a way of substantially strengthening the argument by reference to the explanatory role of attractors in dynamical systems theory (sec. 3). From the modal-dependence view of explanation - which, I will go on to suggest, is a live option with respect to the kinds of explanation at stake - this strengthening fails to shore up the Platonist conclusion (sec. 4). I will conclude with broader reflections regarding explanatory indispensability in connection with a modal perspective on explanation to motivate the broader thesis expounded above (sec. 5).

2. The Explanatory Power of Phase Spaces. Lyon and Colyvan (2007) argue that models in dynamical systems theory, in Hamiltonian classical mechanics in particular, provide an example of explanatory mathematics that can support mathematical realism via EIA. In dynamical systems theory, mathematics can play an indispensable role in the presentation of phase spaces and their explanatory features. Points of a phase space represent a continuously infinite range of possible instantaneous dynamical states of a physical system, and trajectories through these points represent the system's possible time evolutions. In dynamical systems theory, mathematics provides an indispensable means for presenting such modal information and theorizing about it in various ways.

Lyon and Colyvan (2007) emphasize the explanatory import of phase spaces in order to amplify Malament's (1982) objection to Field's (1980) nominalism. Malament challenged Field's program, arguing that Field is not entitled to quantify over possible dynamical states in theories that trade in phase spaces. A nominalist might respond to Malament by shifting focus to an empirically equivalent Lagrangian formulation, which does not rely on phase space structures and hence is more amenable to nominalization à la Field. Against this move, Lyon and Colyvan argue that Hamiltonian mechanics still raises serious issues for the nominalist due to its indispensable explanatory virtues: pointing to an alternative, potentially nominalizable presentation of dynamical systems arguably does not help the nominalist, if there are some features of the world that we can explain only with an account relying on phase spaces.

As an example of such phase space explanation, Lyon and Colyvan point to the Hénon-Heiles model. This is a well-known, very simple, and widely applied model of nonlinear systems that can be used to study and explain chaotic features of various kinds of systems with 2 degrees of freedom. (Hénon and

1. See Woodward (2003) for a modal-dependence view of causal explanations and Reutlinger (2015) for a modal-dependence view of noncausal explanations. 
Heiles used it to model the motion of a star, confined to a plane, around a galactic center.) The Hénon-Heiles Hamiltonian can be written as a function of momenta $p_{x, y}$ and spatial coordinates $x, y$ as

$$
H=\frac{1}{2}\left(p_{x}^{2}+p_{y}^{2}\right)+\frac{1}{2}\left(x^{2}+y^{2}\right)+x^{2} y-\frac{y^{3}}{3} .
$$

In dynamical systems theory, solutions of the equations of motion (derivable from the Hamiltonian) are typically studied via a Poincaré map. ${ }^{2}$ This allows one to study, for example, the relative prominence of chaotic versus regular solutions of the Hénon-Heiles Hamiltonian as a function of the total energy of the system as a control parameter that a modeler can vary (see Hilborn 2000; for Hamiltonian systems energy is constant). Lyon and Colyvan argue that this kind of information about chaotic versus regular solutions can be explanatory and that it would be impossible to extract this information from an alternative Lagrangian description of the same system. Thus, the Hamiltonian formulation, with its use of phase spaces, gains an explanatory edge.

What empirical phenomenon can only be explained in these terms? Lyon and Colyvan point to the fact that for systems well modeled by HénonHeiles equations "high-energy Hénon-Heiles systems tend to exhibit chaotic and unpredictable motion, and low-energy Hénon-Heiles systems tend to exhibit regular and predictable motion" $(2007,237)$. This fact can arguably be explained by the Hénon-Heiles model (studied via the Poincaré map), which shows "how the regions of possible dynamical states that give rise to chaotic motion change as the total energy of the system changes" (237). Thus, the Hamiltonian analysis of such a system can explain and give us understanding not available otherwise: "The explanatory power is in the structure of the phase space and the Poincaré map. So it seems that this is a case where using the phase space is essential to our understanding and ability to explain certain features of the world" (238).

In particular, without the Hamiltonian analysis we do not understand how the structure of the phase space changes - how stable large-scale features of the phase space emerge and grow — as the total energy decreases. It is important to note that the explanandum at stake does not concern any particular trajectory through phase space; it does not concern any particular event or regularity that could be associated with an actual system. Rather, it concerns structural features of phase space: a system's 'tendency' to exhibit regular or chaotic behavior is a matter of how it relates to a relevant class of other possible trajectories. The Poincaré map analysis of the Hénon-Heiles Hamiltonian

2. A Poincaré section is a lower-dimensional subspace of a phase space, transversal to the dynamical flow of the system. A Poincare map is the intersection of a periodic orbit with a Poincaré section. It is a useful tool in analyzing a dynamical system since it preserves many properties of the trajectories of the original system. 
explains this tendency in terms of changes in the relative proportion of chaotic versus regular trajectories, as a function of the system's energy (fig. 1).

Granting the explanatory utility of phase spaces, one may nevertheless question whether mathematics is in any substantial sense involved in the explanation. Admittedly it seems to provide an indispensable means to represent all the possible dynamical states and possible trajectories and, more important, how the properties of the trajectories - being chaotic versus regular - depend on systems' energy. But what seems to matter for the explanation itself, really, is a wealth of information regarding possible trajectories.

Lyon and Colyvan anticipate this move, but they regard it as illegitimate for the nominalist: "points in phase spaces are abstract objects that represent other abstract objects (possible dynamical states)" (2007, 233, my emphasis). A nominalist who wishes to place the explanatory burden on possible dynamical states and trajectories achieves a pyrrhic victory at best: trading quantification over mathematical abstracta for quantification over possibilia cannot save the nominalist day. ${ }^{3}$

How convincing is this rebuttal of the nominalist move? This depends on one's view of the explanation at stake. The rebuttal does not elucidate mathematics' function in the explanation, nor does it show that mathematics is doing any real explanatory work. Prima facie, the explanatory work is done by purported facts about possible systems. Furthermore, the involvement of such modal facts is far from surprising, given that the explanandum itself makes reference to modal features. Recall Lyon and Colyvan's question: why is it that systems well modeled by the Hénon-Heiles Hamiltonian tend to increasingly exhibit chaotic and unpredictable motion at higher energies? This question is not about any actual solution of the Hamiltonian equations of motion. It is about a whole large class of possible solutions. It is not fully clear how a trajectory's 'tendency' to be chaotic/regular should be interpreted exactly, but however it is precisified, it will surely involve reference to nonactual trajectories. Given that the explanandum is thus modally laden, one readily expects the explanans to involve similar modal content as well-hence, the appeal to nonactual trajectories in the explanation. A nominalist should not find this 'explanatory power of possibilia' extraordinary or surprising at all. The nominalist can argue, furthermore, that the Hamiltonian formulation provides an economical way - the only way that is even nearly economical enough for limited beings such as ourselves - to represent the relevant modal features.

3. Similarly, Baker and Colyvan $(2011,328)$ lament: "Mathematical nominalists eschew mathematical objects because of epistemic and other problems associated with admitting them into one's ontology. Avoiding commitment to mathematical objects at the expense of admitting possible worlds is simply not a good deal." It is a contestable issue how quantification over possibilia amounts to antinominalism, however. Indeed, there are welldeveloped modal nominalist accounts of mathematics itself, aiming to eschew commitment to mathematical objects in modal terms (e.g., Hellman 1989; Chihara 1990). 


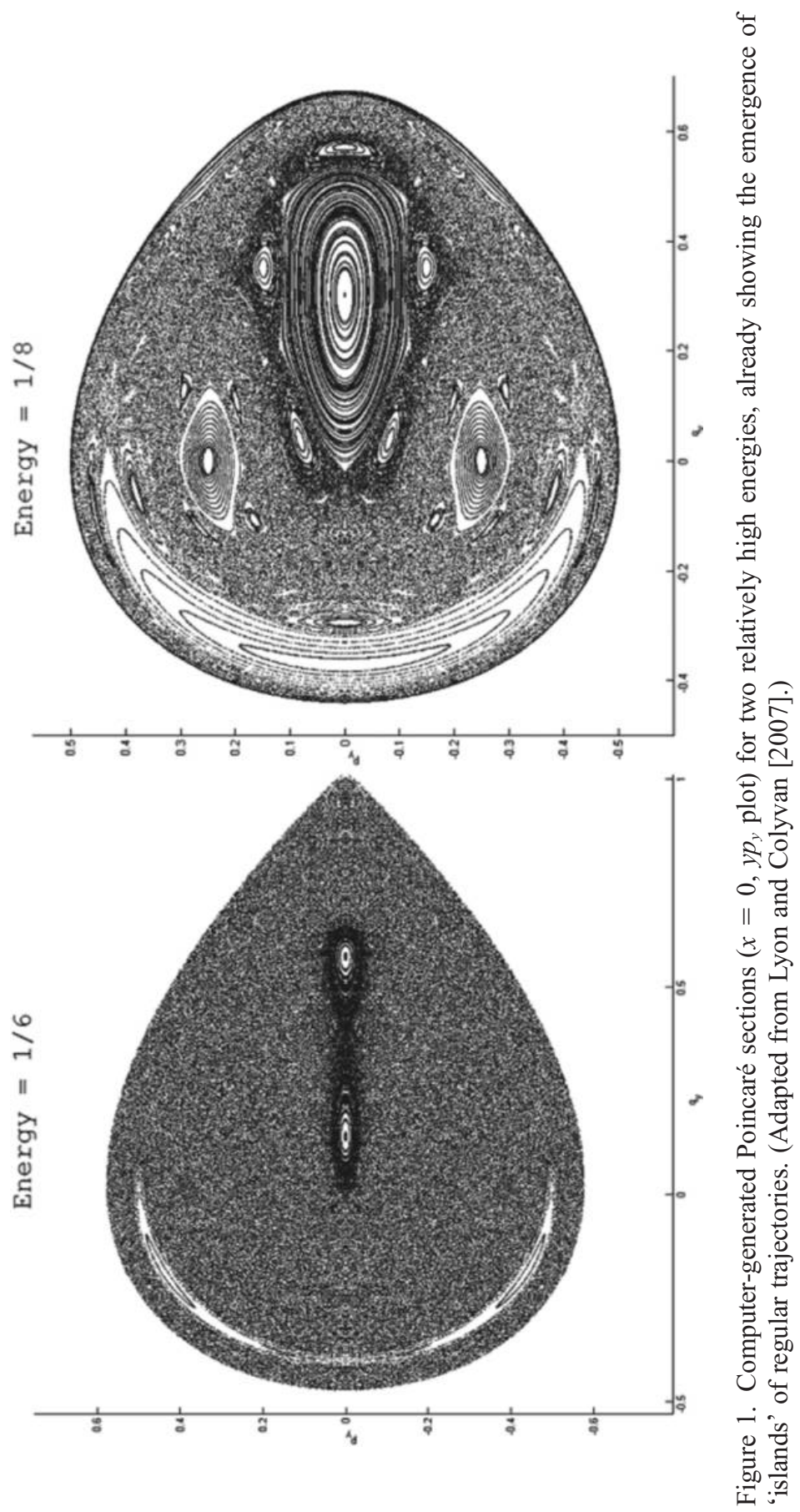

This content downloaded from 129.011.022.236 on July 17, 2018 05:47:02 AM

All use subject to University of Chicago Press Terms and Conditions (http://www.journals.uchicago.edu/t-and-c). 
What about realist commitment to abstract possibilia, then? Can the nominalist get off the hook here? This issue is too large to be fully dealt with here, but let me indicate why the upshot is far from clear. The issue turns on how we should understand the nature of the explanation at stake. Lyon and Colyvan say nothing about this, apart from noting that the explanation is not causal. While they are surely right about this, it is still natural to try to account for the explanation in the spirit of a counterfactual, modal-dependence view of explanation (see Reutlinger 2015; Saatsi 2016b). After all, the explanation clearly provides information of the requisite sort: a trajectory's tendency to be chaotic (or otherwise) depends on the system's energy and its initial conditions (in relation to the area of phase space to which the trajectory belongs - i.e., whether a trajectory is within one of the 'islands' of regular trajectories).

The explanatory analysis of the Hénon-Heiles Hamiltonian in terms of the Poincaré map allows us to grasp explanatorily relevant modal features of the system being represented. Arguably this is how we explain and gain explanatory understanding. As Lyon and Colyvan note, the phase space approach puts us in a position to relate the actual state of affairs, which is represented, in an idealized way, by a particular solution, to a whole network of nonactual possibilities. In the Hénon-Heiles model this amounts to seeing certain coursegrained features of the phase space (e.g., areas of phase space dominated by regular or chaotic solutions) and how these features are altered under changes in the control parameter $E$ (energy). This kind of modal information is naturally construed as the kind of 'what-if-things-had-been-different' information that Woodward and others have emphasized being at the heart of explanatory understanding. Admittedly the changes in the parameter $E$ are not causally connected to the explanandum. But given the nature of the explanandum ('tendency to be chaotic') this is far from surprising. The noncausal nature of the explanatory dependence need not be problematic from the perspective of the counterfactual view of explanation: it has been argued in various other contexts that there are good reasons to liberalize the notion of 'explanatory counterfactual' from its causal connotations and apply the modal-dependence framework beyond causal explanation (see, e.g., Saatsi and Pexton 2013; Reutlinger 2015; Saatsi 2016a).

Thinking about the explanatory capacity of the Hénon-Heiles model in modal terms begins to throw new light on the explanatory virtues of phase space formulation of dynamical systems and the role that quantification over possibilia plays in such explanations. Such quantification may be ontologically committing from the perspective of the deductive nomological model, perhaps, if we assume that explanatory success requires that all the explanans are simply true. From the perspective of modal accounts of explanation, however, there is scope for the nominalist to fully endorse the explanatory importance of modal information. This leads to a natural extension of a popular 'indexing' strategy against indispensability arguments. The gist of the indexing 
strategy is to maintain that mathematics only plays a straightforwardly representational role of indexing physical properties, thereby radically enhancing theories' expressive powers (e.g., Melia 2000; Daly and Langford 2009). Consider, for instance, the representational power of mathematical Euclidean space $\mathbb{R}^{3}$ as indexing numerous physical facts about spatial distances, angles, and so on. The indexing strategy needs to be extended here, however, since the modal information involved in the Hénon-Heiles model concerns nonactual states and structures thereof: the mathematical points and structures of phase space must be viewed as indexing representations of (nonactual) possibilities regarding physical variables. The modal information gained through such representations can be explanatory vis-à-vis an actual trajectory by virtue of providing appropriate kinds of modal information about explanatory dependencies.

As said, Lyon and Colyvan anticipate and object to this move as involving an illegitimate appeal to possibilia. They dismiss the 'modalized' indexing maneuver too quickly, however. For even if this move does not win the war, it might still win the battle, so to speak. First of all, from the standpoint of modal accounts of explanation one expects modal information concerning nonactual states of affair to feature centrally in scientific explanations. This makes clear the explanatory virtue of mathematical representations of dynamical systems that are geared toward displaying the relevant kind of modal information. Second, the nominalist admittedly needs to offer an account of the relevant explanatory counterfactuals that these representations inform us about. But this is a different battle, one that the nominalist faces in any case, independently of phase space theories. Science is full of representations of nonactual states of affairs that are absolutely indispensable for explaining, and examples of mathematical explanations add little to the pressure the nominalist should already feel to account for these representations, if we can regard these mathematical explanations merely as indexing such nonactual states of physical affairs.

Lyon and Colyvan's phase space argument for Platonism thus seems inconclusive at best: it is not enough for (mathematical) EIA to point to explanatorily indispensable modal facts. However, dynamical systems theory provides interesting opportunities to push further the dialectic between the Platonist and the nominalist, as I next discuss.

3. The Explanatory Power of Attractors. The Hénon-Heiles model is but an isolated example of dynamical systems theory in explanatory action, and a broader look at a variety of explanations offered by analytical models of dynamical systems suggests an alternative focus for the Platonist argument. In particular, it allows the Platonist to zoom in on explanations that essentially involve mathematics that seemingly cannot be interpreted as representing (or 'indexing') anything physical at all, actual or otherwise. 
A candidate for such nonrepresentational explanatory mathematical constructs are phase space attractors: sets of points of the phase space of a dynamical system (identified by its equations of motion) that are long-term stable, that is, points toward which the system's dynamical behavior is attracted after shorter-term behavior has died down. Attractors are abstract mathematical objects, but their geometrical, topological, and other properties can reveal explanatory information about a system's dynamical behavior. A simple damped harmonic oscillator has a fixed point as its attractor, while a dissipative anharmonic oscillator can have a periodic limit cycle attractor, for example. Of special interest are strange attractors: aperiodic limiting solutions of a dissipative system toward which all solutions (within a basin of attraction) asymptotically approach. (The Lorenz attractor is a well-known example from nonlinear systems theory.)

In dynamical systems theory, attractors are used to explain various qualitative and quantitative features of long-term dynamic behavior (see, e.g., Strogatz 1994; Hilborn 2000). For example, features of attractors can explain how chaotic systems can from very different starting points tend toward behavior that is similar with respect to those features, despite the trajectories being at the same time highly sensitive to initial conditions. A dissipative dynamical system can exhibit coarse-grained features of a 'two-winged' Lorenz attractor in the long term, for example, despite starting from very different places in the phase space (within the basin of attraction). ${ }^{4}$ The onset of chaos in a deterministic system is also standardly explained in terms that appeal to attractors and their sudden qualitative change ('bifurcation') under a change in a control parameter. Also, the universality of a particular way in which microphysically very different systems start exhibiting chaotic behavior (e.g., via period doubling) is explained in terms of attractors. Long story short: strange attractors are indispensable for explaining various features of nonlinear dynamical systems. (For detailed examples, see Strogatz [1994] and Hilborn [2000]. For philosophical discussion, see Smith [1998].)

Despite their explanatory power, there are very good reasons to not take strange attractors to represent a specific set of possible initial conditions of a dynamical system. The issue is that these attractors have an infinitely finegrained, self-similar fractal structure that cannot correspond to the structure of possible states of physical systems individuated with much more coarsegrained dynamical properties. As Smith (1998) clearly explains, strange attractors play an indispensable explanatory role with respect to nonlinear systems with dynamical quantities such as fluid circulation velocity, temperature, the proportional concentration of a chemical in a mixture, and so on. These are macroscopic, 'averaged' quantities that clearly cannot have indefinitely precise

4. The basin of attraction is defined as the set of phase space points that are attracted toward an attractor in the long term. 
real number values required for the structure of a strange attractor. Averaged properties such as temperature at a point $P$ in a fluid simply cannot have perfectly determinate values, even notwithstanding the fact that 'zooming in' the physical structure fluid at some point gives rise to the quantum world (see Smith 1998, chap. 3). Yet it is the dynamics of these kinds of quantities that strange attractors are used to explain.

In response to this puzzle he raises, Smith (1998) argues that strange attractors are mere mathematical by-products of a theoretical description that latches onto more robust, more coarse-grained dynamical features of physical systems. ${ }^{5}$ Koperski (2001) argues in a similar vein that strange attractors are indispensable idealizations, mathematical by-products of the fact that we must use differential equations in the mathematical modeling of dynamical systems needed to explain their dynamical behaviors. I am sympathetic to this way of thinking about attractors, but it is worth noting that neither of these authors provides any philosophical account of the explanations in which attractors thus figure. ${ }^{6}$ Furthermore, such an account is needed in order to see what we should make of the explanatory indispensability of attractors vis-à-vis EIA, in order to not simply beg the question against an advocate of EIA. I will next suggest that a modal-dependence view provides a natural way to account for the key features of these explanations. This matters in the context of EIA, since the ontological commitments of strange attractors are not at all obvious from this modal perspective, as we will see by reflecting more generally on realist commitments of the modal-dependence view of explanation.

4. Explanatory Dependencies in Dynamical Systems. As we have seen, the explanatory role played by attractors provides an interesting opportunity to push further the Platonist argument, since the 'modalized' indexing move is no longer an option: attractors do not as such represent anything physical, actual or otherwise. There is a different response the nominalist can make here, however. The nominalist can maintain, building on Smith (1998) and Koperski (2001), that explanatory heavy lifting in the study of chaotic systems is done by features of the mathematical models that are much less fine grained than attractors themselves. The latter are to be regarded as mere mathematical by-products of models that are explanatory by virtue of latching onto appropriate modal facts, captured by explanatory regularities concerning changes in more coarse-grained aspects of a target system as a function of a control parameter.

5. In particular, for Smith a strange attractor is a sign of a 'stretching-and-folding' process of trajectories that result in fractals only if taken to the infinite limit. The stretchingand-folding processes latch onto an actual system in an explanatory way.

6. Providing such an account is an open philosophical issue. Bishop (2015) very briefly toys with some options. 
Consider the following Lorenz equations, for instance.

$$
\begin{aligned}
& \frac{d x}{d t}=10(y-x), \\
& \frac{d y}{d t}=r x-y-x z, \\
& \frac{d z}{d t}=x y-\frac{8 z}{3} .
\end{aligned}
$$

Solutions of these equations can behave in radically different ways in their coarse-grained features for different values of the adjustable parameter $r$. When these equations are used to model the well-known phenomenon of Rayleigh-Bènard convection, for example, $r$ can be identified as being directly proportional to the constant temperature difference between two parallel surfaces containing the convection. In an experimental setting, this temperature difference (and hence $r$ ) is a control parameter that can be manipulated to change the nature of the convection. Lorenz equations, and their associated two-winged Lorenz attractor, can thus be used in explaining (and predicting) features of Rayleigh-Bènard convection by capturing the dependencies of various coarsegrained aspects of the physical phenomenon on the temperature difference (see Smith 1998, sec. 4.5, for discussion).

The Lorenz attractor, which represents, for example, the long-term shape and general location of phase space trajectories of the relevant state variables, can itself be regarded as an idealization and mathematical surplus. But this does not take away the fact that this mathematical construct is an indispensable component of best explanations of the chaotic behavior of Rayleigh-Bènard convection. And it does not mean that attractors' explanatory indispensability should elicit realist commitment to either the mathematics involved or some feature of the world directly represented by them (with respect to either actual or possible systems, as the 'indexing' move would have it). This is because there are other ways to understand attractors' role in explanations.

The modal-dependence view on explanation, in particular, can help the nominalist to gain the upper hand in the dialectic against the Platonist. From this perspective we can regard the power of explanations of chaotic behavior as residing in information about modal dependencies between more coarse-grained, physically well-defined variables. Explanations of real world systems in terms of models featuring strange attractors do not relate any explanandum variable to the infinitely fine-grained features of the attractor: as far as explanatory models of nonlinear systems are concerned, no feature of physical systems depends on the fine-grained features of attractors. Rather, the explanatory dependences hold between more coarse-grained features that are naturally represented by coarse-grained features of the attractors. For example, explanations of chaotic behavior often capture the way in which abrupt changes (bifurcations) in the 
more coarse-grained features take place in response to a change in a control variable that can typically be regarded as a (broadly speaking) manipulable variable in the spirit of Woodward (2003). Since the modeling of these dynamical systems requires differential equations, the mathematics automatically leads to geometrical structures like strange attractors that then naturally obtain a representational role with respect to the more coarse-grained features of phase space that are modally connected to other variables in an explanatory way.

5. Broader Reflections. I have argued elsewhere in much more general terms that explanations' realist commitments are best understood and analyzed in relation to well-formed ideas about what it is to explain (Saatsi 2016b). This commonsensical point has been ignored in debates about explanatory indispensability for too long. The reason why well-formed ideas about explanation matter is that in modal accounts of explanation, for example, explanatory indispensability turns out to be insufficient in and of itself for justifying ontological commitment. I have tried to explicate this in general terms in Saatsi (2016b), through a distinction between different kinds of explanatory roles: some 'thick' and ontologically committing, others 'thin' and ontologically peripheral. This is a way to reconcile the idea that mathematics can indispensably contribute to an explanation yet at the same time not be ontologically committing.

Here I have argued in this spirit that the modal-dependence perspective on the explanations furnished by dynamical systems theory can also undermine the argument from Lyon and Colyvan (2007), even when this argument is strengthened with reference to features of dynamical systems theory — strange attractors - that cannot be dealt with by the standard nominalist strategy of 'indexing'.

\section{REFERENCES}

Baker, A. 2009. "Mathematical Explanation in Science." British Journal for the Philosophy of Science 60 (3): 611-33.

Baker, A., and M. Colyvan. 2011. "Indexing and Mathematical Explanation." Philosophia Mathematica 19 (3): 323-34.

Bishop, R. 2015. "Chaos.” In Stanford Encyclopedia of Philosophy, ed. Edward N. Zalta. Stanford, CA: Stanford University. https://plato.stanford.edu/entries/chaos/.

Chihara, C. 1990. Constructibility and Mathematical Existence. Oxford: Oxford University Press.

Daly, C., and S. Langford. 2009. "Mathematical Explanation and Indispensability Arguments." Philosophical Ouarterly 59 (237): 641-58

Field, H. 1980. Science without Numbers. Oxford: Oxford University Press.

Hellman, G. 1989. Mathematics without Numbers: Towards a Modal-Structural Interpretation. Oxford: Clarendon.

Hilborn, R. C. 2000. Chaos and Nonlinear Dvnamics: An Introduction for Scientists and Engineers. Oxford: Oxford University Press.

Koperski, J. 2001. "Has Chaos Been Explained?" British Journal for the Philosophy of Science 52 (4): $683-700$.

Lyon, A. 2012. "Mathematical Explanations of Empirical Facts, and Mathematical Realism." Australasian Journal of Philosophy 90 (3): 559-78.

Lyon, A., and M. Colyvan. 2007. "The Explanatory Power of Phase Spaces." Philosophia Mathematica 16 (2): $227-43$. 
Malament, D. 1982. "Science without Numbers by Hartry H. Field." Journal of Philosophy 79 (9): $523-34$.

Melia, J. 2000. "Weaseling Away the Indispensability Argument.” Mind 109 (435): 455-80.

Psillos, S. 2010. "Scientific Realism: Between Platonism and Nominalism." Philosophy of Science 77 (5): 947-58.

- 2011. "Living with the Abstract: Realism and Models." Synthese 180 (1): 3-17.

Reutlinger, A. 2015. "Is There a Monist Theory of Causal and Non-causal Explanations? The Counterfactual Theory of Scientific Explanation." Philosophy of Science 83:733-45.

Saatsi, J. 2016a. "On Explanations from Geometry of Motion." British Journal for the Philosophy of Science. doi:10.1093/bjps/axw007.

- 2016b. "On the Indispensable Explanatory Role of Mathematics.” Mind 125 (500): 1045 70. doi:10.1093/mind/fzv175.

Saatsi, J., and M. Pexton. 2013. "Reassessing Woodward's Account of Explanation: Regularities, Counterfactuals, and Noncausal Explanations.” Philosophy of Science 80 (5): 613-24.

Smith, P. 1998. Explaining Chaos. Cambridge: Cambridge University Press.

Strogatz, S. H. 1994. Nonlinear Dynamics and Chaos: With Applications to Physics, Biology, Chemistry, and Engineering. Cambridge, MA: Perseus.

Woodward, J. 2003. Making Things Happen: A Causal Theory of Explanation. Oxford: Oxford University Press. 